\title{
Género Trichosporon
}

\author{
Trichosporon genus
}

Generalidades. Trichosporon sp son levaduras ubicuas y generalmente se encuentran en sustratos ambientales y madera descompuesta. Forman parte de la microbiota comensal intestinal en el ser humano y colonizan de manera transitoria la piel y la vía respiratoria. El género Trichosporon fue creado en 1890 y aislado de micosis superficiales en humanos. Además se ha aislado de procesos infecciosos profundos e invasores. Por muchos años se conoció sólo una especie saprófita asociada a la piedra blanca, denominada T. beigelli. Actualmente, en base al análisis molecular, este taxón ha sido reemplazado por varias especies y la taxonomía del género se ha modificado.

Taxonomía. Tradicionalmente Trichosporon se clasificaba de acuerdo a características morfológicas, ecológicas y fisiológicas, pero, con resultados inconsistentes, por lo que la especie fue reclasificada en base a estudios filogenéticos con herramientas de biología molecular. Las especies clásicamente aisladas en procesos patológicos son T. cutaneum, T. asahii, T. asteroides, T. mucoides.
T. inkin y $T$. ovoides. Se han descrito más de 36 especies, agrupadas en el orden Trichosporonales y en los clados Gracile, Porosum, Cutaneum y Ovoides.

Importancia médica. En general, estas especies se asocian a infecciones benignas superficiales, particularmente a la piedra blanca, la cual, es producida por T. inkin (vello púbico) y $T$. ovoides (cabello). Pueden producir onicomicosis (T. cutaneum), recuperarse de tinea pedis y de lesiones anales en pacientes con infección por VIH y piedra genitourinaria. Se han descrito cuadros de neumonía alérgica ( $T$. asahii) y neumonitis alérgica del verano por inhalación de los artroconidios asociados al calor y la humedad en Japón en esa estación. Las infecciones invasoras por Trichosporon son precedidas, comúnmente, por una colonización respiratoria o gastrointestinal y con frecuencia se asocian al uso de catéter venoso central. Representan la segunda causa de infección fúngica invasora por levaduras en hospederos inmunosuprimidos. Producen fungemia en pacientes con neutropenia y cán- cer, en forma similar a la candidiasis. Se ha aislado desde abscesos (cerebral, hepático), endocarditis, peritonitis y asociado a fracaso terapéutico por resistencia microbiológica de algunas especies a anfotericina B. También se han descrito casos de infecciones oftalmológicas post cirugía, infecciones de prótesis, de peritoneo-diálisis, meningoencefalitis y neumonía en inmunocompetentes, quedando en evidencia un rol patogénico de esta especie.

Características microbiológicas e identificación. Trichosporon, al igual que Geotrichum, son capaces de formar artroconidios (Figura 1). Una característica que diferencia a ambas especies es la capacidad de hidrolizar la urea; Trichosporon la hidroliza. Aunque ambas especies son similares fenotípicamente, difieren mucho genotípicamente. Trichosporon es de crecimiento rápido en cultivo y las colonias se presentan con un aspecto céreo y blanquecino (Figura 2). La identificación bioquímica de especie es usualmente difícil, con problemas de reproducibilidad y precisión. Algunas prue-

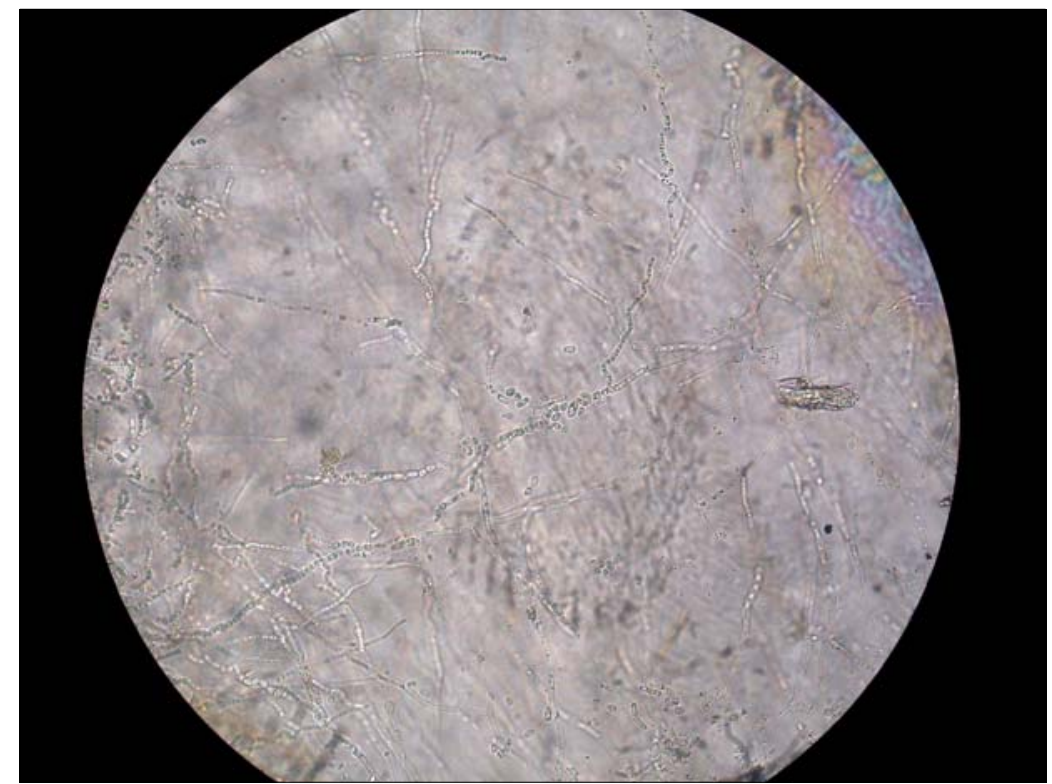

Figura 1. Microcultivo de Trichosporon sp. Las flechas negras muestran artroconidios.

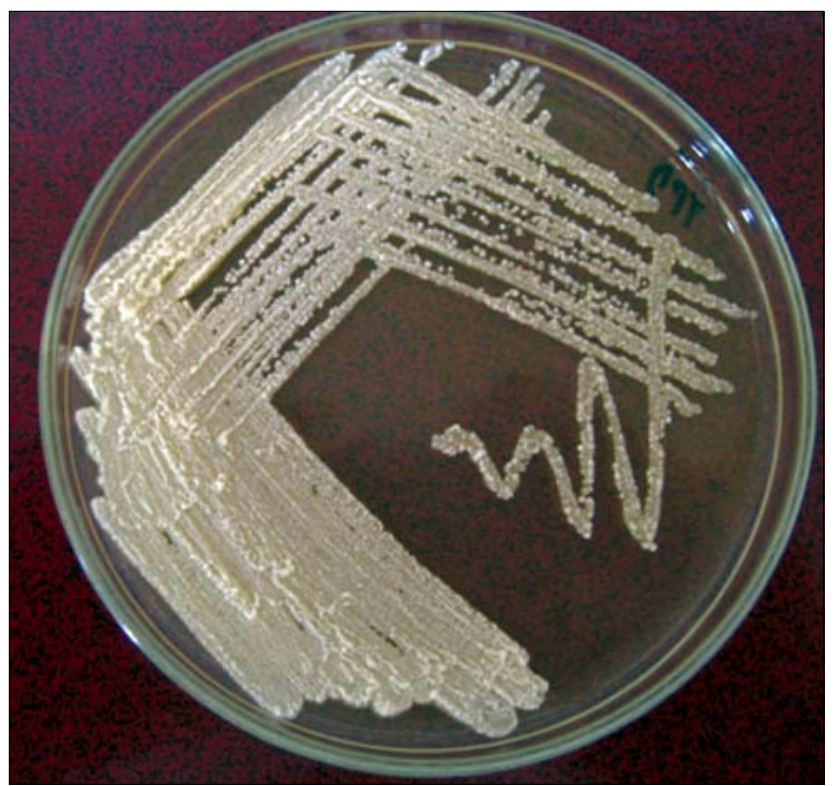

Figura 2. Colonia de Trichoporon sp. 
bas incluyen asimilación de L-arabinosa, sorbitol, melibiosa y mio-inositol, crecimiento a $37^{\circ} \mathrm{C}$, crecimiento en presencia de cicloheximida al $0,1 \%$ y formación de apresorios (estructuras observadas en microcultivo). Actualmente existen kits manuales y para equipos automatizados disponibles en el mercado; sin embargo, estos métodos no incorporan las nuevas categorías taxonómicas. Cada vez más, se utilizan métodos basados en amplificación o secuenciación de regiones específicas del ADN ribosomal de Trichosporon que incluyen, RPC de la región 18S, secuenciación de ITS (internal transcriber spacer), secuenciación de IGS (intergenic spacer regions) y detección de regiones 26S (D1/D2), de ITS y de IGS mediante sondas cuya señal es detectada con citometría de flujo.

Susceptibilidad in vitro. A pesar de que algunas infecciones por Trichosporon son refractarias al tratamiento antifúngico convencional, pocos estudios han evaluado nuevos compuestos. El documento para susceptibilidad antifúngica del CLSI no incluye al género Trichosporon. Autores europeos han utilizado la metodología del European Committee for Antimicrobial susceptibility Testing (EUCAST) en Trichosporon a pesar de que ésta ha sido estandarizada sólo para levaduras fermentadoras de la glucosa (Candida sp). Cabe señalar, eso sí, que determinadas especies, como $T$ asahii, $T$. faecale y $T$. coremiiforme presentan resistencia in vitro a anfotericina $B$. Los azoles tienden a ser activos frente a la mayoría de las especies de Trichosporon, siendo el más potente, voriconazol.

Conclusiones. Trichosporon es una levadura que produce infecciones superficiales benignas y puede producir infecciones invasoras en pacientes inmunocomprometidos; en determinadas circunstancias, causa infecciones profundas en inmunocompetentes. La identificación por métodos convencionales presenta problemas, siendo la mejor alternativa para estos propósitos el uso de herramientas moleculares, disponibles en laboratorios especializados. Debido a que existen diferencias en la susceptibilidad antifúngica de las especies, resultaría más práctico solicitar este tipo de estudio previo a la identificación de especie, con el fin de ajustar rápidamente la terapia antifúngica. Las dificultades diagnósticas y terapéuticas relativas a Trichosporon hacen que este agente aún constituya materia de estudio.

\section{Referencias}

1.- Chagas-Neto T C, Chaves G M, Colombo A L. Update on the genus Trichosporon. Mycopathologia 2008; 166: 121-32.

2.- Rodríguez-Tudela J L, Díaz-Guerra T M, Mellado E, Cano V, Tapia C, Perkins A, et al. Susceptibility patterns and molecular identification of Trichosporon species. Antimicrob Agents Chemother 2005; 49: 4026-34.

Cecilia Tapia $\boldsymbol{P}$.

Laboratorio de Micología Médica. Programa de Microbiología y Micología, ICBM. Facultad de Medicina de la Universidad de Chile cetapia@med.uchile.cl 